\title{
Apparel-Oriented Anthropometric Database of Colombian Military Personnel
}

\author{
David MONTANO, Ana María POLANCO, Alejandro MARANON* \\ Department of Mechanical Engineering, Universidad de Los Andes, Bogota, Colombia
}

\begin{abstract}
This work addresses the need for developing an anthropometric database of the Colombian military personnel, as a part of the ongoing project "Composite materials for ballistic protections systems", funded by the Military Industry of Colombia (INDUMIL). In this paper are presented the results obtained in the first stage of the database construction, in compliance with international standards ISO 15535 and ISO 8559.

In this phase, a total of 99 subjects were scanned using a $[T C]^{2}$ NX-16 Full Body Scanner. The 3D body models obtained allowed the extraction of 44 anthropometric parameters, which were processed and reported using descriptive statistics. A quality control procedure was implemented to identify outlying values corresponding to defective body models. A validation procedure is proposed based on the assumption that body dimensions are distributed normally.

All but one of the data sets were found to follow the standard Gaussian Distribution at the 95\% confidence level, which is a good indicator of the consistency of data extraction and processing among all subjects.

A statistical description of the colombian military population has never been reported in the literature, therefore the military industry is lacking of very important information for addressing the needs of fit, comfort and coverage that protective equipment must deliver to military personnel. This work is intended to be the first of its kind for colombian population, and it will be a very powerful tool for designers and military garment developers, which will use reliable data about the anthropometric dimensions of their target population.
\end{abstract}

Keywords: Anthropometric database, Full body scanning, Quality control and data validation.

\section{Introduction}

Due to the effortless access that criminal organizations have to firearms, wearing of protective equipment against ballistic threats has become essential for military personnel and law enforcement officers safety. This kind of garment has functional attributes given by the mechanical properties of certain materials that, by virtue of their capability of defeating projectiles and mitigate blunt trauma, are known as ballistic materials.

Panels made of ballistic materials layers are capable of absorbing or redistributing the impact energy transferred from a projectile to the body, so is dispersed before perforation of the material and penetration of the bullet through the skin. The kinetic energy is dissipated due to the work absorbed during panel deformation or fracture. Different materials and configurations are employed according to the amount of energy of the ballistic threat meant to be defeated, so features like weight, flexibility and thickness of the ballistic panels vary in detriment of user comfort, for higher levels of protection.

In this context, one of the most important protective systems are ballistic vests or body armors, which cover the wearer's torso, providing protection for vital organs such as the heart, lungs and liver. The armor incorporates pockets that allow the ballistic panel or panels to be placed on several positions on the garment, giving different configurations depending on the level of coverage desired.

A body armor consists of a panel that provides the ballistic protection and an armor carrier, that retains the panel and provides means of securing the garment to the user.

The most basic level of coverage is a carrier with two pockets: one on the front and on the backside; nevertheless, the coverage often extends from the torso to sensitive, high vascularized areas like the neck and the crotch, in order to reduce the risk of hemorrhage due to ballistic wounds. As expected, a higher level of coverage compromises the mobility and comfort of the user, so a delicate balance must exist between all design criteria to obtain the best relation of all the variables involved.

*emaranon@uniandes.edu.co 
For a soldier patrolling during extended periods of time, under the rough environmental conditions of the tropical jungle, comfort and fit are very relevant issues that must be taken into consideration. Otherwise, no matter how good the ballistic panel comes out to be, the protection will not be adequate. These are some of the scenarios related with the ergonomics of the armor that result in an improper protection:

- Soldiers using uncomfortable body armors, remove them because they interfere with their maneuvers.

- Body armor does not fit the dimensions and contours of the body, therefore the coverage is inappropriate.

- The soldier fails to concentrate on his maneuvers due to the discomfort generated, hence he exposes himself to a greater risk.

In military research and development, anthropometric data has been widely used in order to provide both objective design criteria for determining shapes, and settling on sizing systems for garment and protective equipment [1]. An anthropometric database provides a standardized statistical description of a given population, and becomes a very powerful tool for addressing the needs of optimal fit and user comfort.

Human-system interfaces must be treated as objectively as interactions between pieces of hardware. It is not acceptable to make assumptions about the physical features of the target population, neither using anthropometric data obtained from dissimilar sources. Here lies the importance of creating an anthropometric database for colombian military personnel, which could provide reliable and statistically significant information about the body dimensions and their variability. Colombian military industry is lacking of information that could be useful for various purposes apart from garment production, among others, for the construction of facilities and work stations, and in conducting comparative anthropometric studies, which are very relevant in the physical anthropology field.

Given its high accuracy, repeatability and speed, a full body scanner was selected over traditional anthropometric methods. The scanner allows to acquire a 3D model of the subject, and it's body dimensions. From the subject's point of view, this is a more private and comfortable method to use due to the lack of contact between him and the anthropometrist. From the researcher's point of view there is a wide number of advantages, as follows:

- This method requires no training on manual measurements techniques, hence human error is reduced.

- High accuracy and repeatability.

- The list of anthropometric variables can be modified and extended after obtaining the 3D model.

However, there are some limitations related with the use of the scanner, basically related to the fact that it is a new technology. Some authors [2-3] have made comparisons between traditional anthropometry methods and 3D scanning methods, concluding there is a need for a new standard of body measurements due to the scanner impossibility of locating various body landmarks which traditionally must be palpated and marked on the body surface.

In the colombian scenario, there are other issues related with the lack of anthropometric studies that inhibit the comparisons with previous results and perform validation studies. Being the body scanning technology such a novelty in this country, where there are no anthropometric studies (not even traditional) conducted on the military personnel, there is a need of validating the study and performing a strong quality control on the data.

This paper presents the results of the research first stage: the creation of an anthropometric database of the colombian military personnel. In this phase, the content of the database was defined according to international standards along with a quality control procedure. A subset of the calculated sample size was scanned and measured, which allowed the authors to gain experience about the use of the scanner. The errors identified during the data acquisition and processing will be very valuable when scanning of the rest of the subjects.

A method for validating the study is also presented. This method has proven to be effective for both quality control of the scans, and for ensuring that the measurement extraction algorithms are consistent among all the subjects. 


\section{Method}

\subsection{Selection of anthropometric variables}

The set of anthropometric variables was determined based on a review of international standards, as follows:

- International Standard ISO 8559: Garment construction and anthropometric surveys - Body dimensions [4].

- International Standard ISO 7250: Basic measurements for technological design - Body measurement definition and landmarks [5].

- International Standard ISO 15535: General requirements for establishing anthropometric databases [6].

- International Standard ISO 20685: 3D scanning methodologies for internationally compatible anthropometric databases [7].

Also, a very important reference work is the military handbook DOD-HDBK-743A [1] (Anthropometry of military U.S personnel). This document is a collection of data acquired over several anthropometric surveys, which provides a complete statistical description for each one of the anthropometric variables. The following list of anthropometric variables was found to be similar to the variables measured in traditional tailoring and garment pattern generation.

Table 1. List of anthropometric measurements

\begin{tabular}{|c|c|c|c|}
\hline \multicolumn{4}{|c|}{ ISO 8559 REFERENCE NUMBER AND NAME } \\
\hline$\underline{2,1,13}$ & UPPER ARM GIRTH [mm] & $\underline{2,2,7}$ & ANKLE HEIGHT [mm] \\
\hline$\underline{2,1,14}$ & ELBOW GIRTH [mm] & $\underline{2,2,9}$ & SCYE DEPTH [mm] \\
\hline$\underline{2,1,15}$ & WRIST GIRTH [mm] & $\underline{2,2,10}$ & CERVICAL TO WAIST [mm] \\
\hline$\underline{2,1,18}$ & THIGH GIRTH [mm] & $\underline{2,2,11}$ & CERVICAL TO KNEE HOLLOW [mm] \\
\hline$\underline{2,1,19}$ & MID THIGH GIRTH [mm] & $\underline{2,2,12}$ & CERVICAL HEIGHT [mm] \\
\hline$\underline{2,1,20}$ & KNEE GIRTH [mm] & $\underline{2,2,13}$ & CERVICAL TO BREAST POINT [mm] \\
\hline$\underline{2,1,21}$ & LOWER KNEE GIRTH [mm] & $\underline{2,2,14}$ & CERVICAL TO WAIST[mm] \\
\hline$\underline{2,1,22}$ & CALF GIRTH [mm] & $\underline{2,2,15}$ & NECK SHOULDER POINT TO BREAST POINT [mm] \\
\hline$\underline{2,1,23}$ & MINIMUM LEG GIRTH [mm] & $\underline{2,2,16}$ & FRONT WAIST LENGTH [mm] \\
\hline$\underline{2,2,2}$ & TRUNK LENGTH [mm] & $\underline{2,2,17}$ & WAIST TO HIPS [mm] \\
\hline$\underline{2,2,3}$ & WAIST HEIGHT [mm] & $\underline{2,2,18}$ & TRUNK CIRCUMFERENCE [mm] \\
\hline$\underline{2,2,4}$ & HIP HEIGHT [mm] & $\underline{2,2,19}$ & CROTCH LENGTH [mm] \\
\hline$\underline{2,2,5}$ & BODY RISE [mm] & $\underline{2,2,20}$ & ARMSCYE GIRTH [mm] \\
\hline$\underline{2,2,6}$ & KNEE HEIGHT [mm] & $\underline{2,2,21}$ & UPPER ARM LENGTH [mm] \\
\hline$\underline{2,2,22}$ & ARM LENGTH [mm] & $\underline{2,2,25}$ & OUTSIDE LEG LENGTH -OUTSEAM [mm] \\
\hline$\underline{2,2,23}$ & CERVICAL TO WRIST LENGTH [mm] & $\underline{2,2,26}$ & THIGH LENGTH [mm] \\
\hline $2,2,24$ & UNDER ARM LENGTH [mm] & $\underline{2,2,27}$ & INSIDE LEG LENGTH [mm] \\
\hline
\end{tabular}

\subsection{Sample size determination}

The sample size must be determined to guarantee the statistical significance of the study. Generally, anthropometric data for technological design are of interest at the $5^{\text {th }}$ and $95^{\text {th }}$ percentile.

The minimum number of randomly sampled subjects, needed to ensure that a database estimates the $5^{\text {th }}$ and $95^{\text {th }}$ percentiles of the population, with $95 \%$ confidence, is calculated using the following equation:

$$
N=\left(\frac{1.96 * C V}{a}\right)^{2} * 1.534^{2}
$$

where:

1.96 is the critical value ( $z$ value) from a standard normal distribution for a $95 \%$ confidence interval; $\mathrm{CV}$ is the coefficient of variation, as follows: 


$$
C V=\frac{S D}{\bar{x}} * 100
$$

where $\bar{x}$ is the arithmetic mean and SD is the standard deviation of the population for the body dimension under study.

Finally, $a$ is the percentage of relative accuracy desired.

In practice, the mean and standard deviation of the population are generally unknown, so these values are estimated using the results from previous similar studies. For this study mean and standard deviation of the population were assumed to be those reported on the military DOD-HDBK-743A, even though it is a different population. This implies hat the variability of anthropometric data within both populations (U.S and colombian military) are similar enough to calculate a satisfactory sample size. Variables with large CV were chosen in order to ensure that the percentiles obtained predict the sample size with the accuracy and confidence desired. Applying Eq. (1), with a percentage of relative accuracy $a=5 \%$, and the CV for each one of the variables, a different sample size was calculated for all the anthropometric items reported on DOD-HDBK-743A.

Among relevant anthropometric data with large $\mathrm{CV}$, it was found for this study that the posterior neck length was the critical variable to be selected $(C V=16.7)$. The sample size calculated was 505 subjects.

\subsection{Materials and Methods}

\subsubsection{Equipment}

The scanner used in this study to acquire the anthropometric variables was de NX-16 developed by $[\mathrm{TC}]^{2}$. This scanner allows the collection of $3 \mathrm{D}$ digital reconstructions of the body surface, capturing between 600.000 and $1 ' 000.000$ points with different spatial coordinates, which provides the digital representation of the scanned subject [8]. From this digital model the extraction of body dimensions was performed with great accuracy and repeatability.

Facilities are shown in Figure 1.

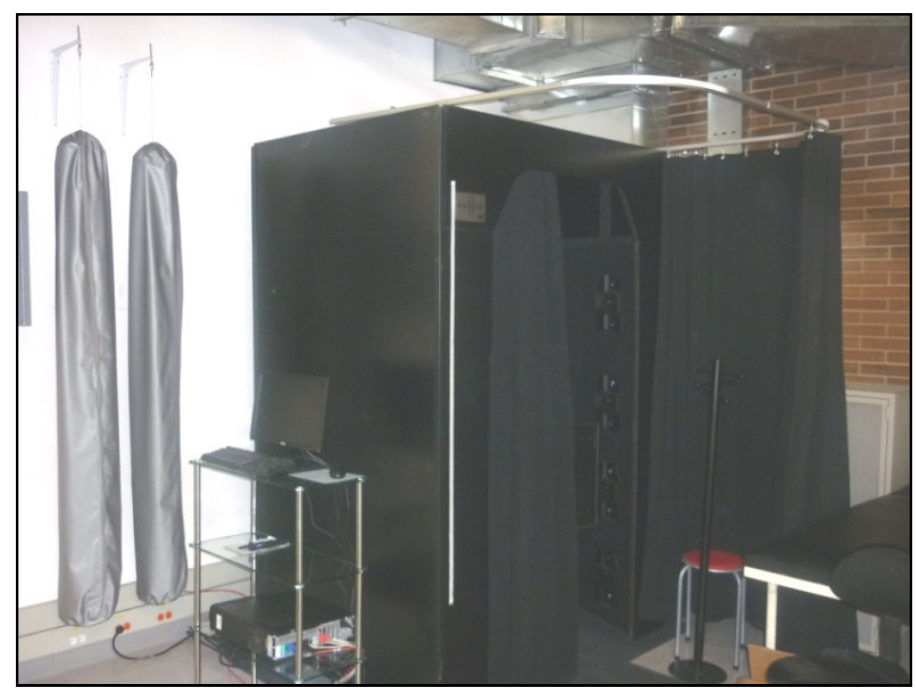

Fig.1. Picture of the NX-16 Body Scanner

\subsubsection{Clothing}

Subjects were provided with grey colored swimming caps and underwear in order to improve light reflection from head and hips region. (See Figure 2) 


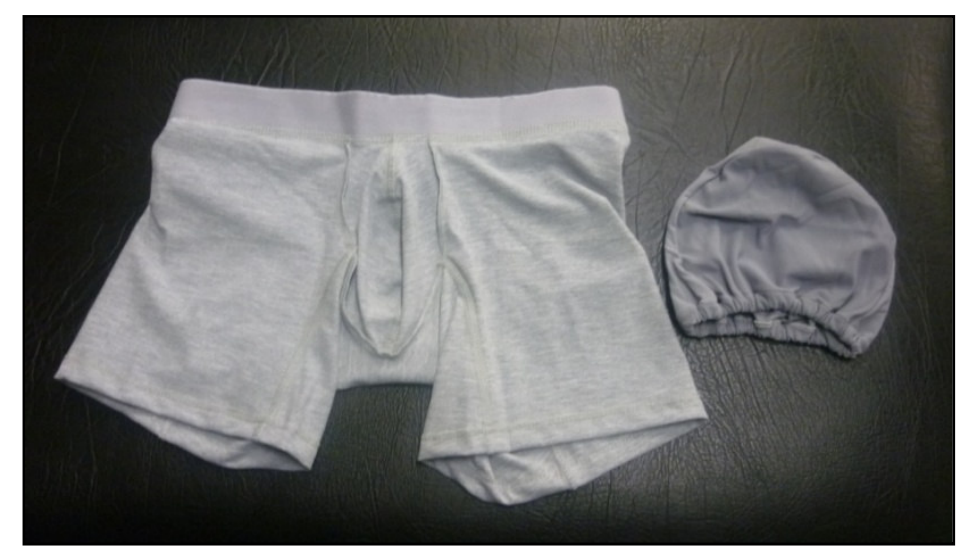

Fig. 2. Clothing provided to the subjects

\subsection{Informed consent and data acquisition}

In 2006, Radkte [9] studied the risk of photosensitivity seizures due to exposition to flashing lights when scanning subjects using the NX-12 system, the previous version of the NX-16. He estimated that 1 in 600.000 healthy adults exposed to the NX12 system would experience an epileptic seizure. Even though this is a very small risk, and that occurrence of a seizure due to the of the scanner has never been reported, it was considered important to inform all the subjects about this possibility.

Confidentiality of the subjects was guaranteed, separating personal information from anthropometric data. The anthropometric database consisted only of the values for each measurement variable. Subjects were aware of the purpose of the study, and authorized the use of their anthropometric measurements in the database.

This was achieved through an informed consent document, containing a brief description of the objectives, procedures, benefits and risks of the study. Once the informed consent letter was read and completely understood, the subject signed it and by this he stated their free will of participating in the study.

After the informed consent letter was signed, each subject filled out a form with their personal background data, such as: Name and last name, date of birth, place of birth, military service time and unit.

They were also asked to provide their shirt and pants size, for further correlation between actual body dimensions and current sizing. Once the documentation was completed, the subject was given the clothing (cap and underwear), and was instructed on how to place himself inside the scanner.

Like shown in Table 1, most of the measurement items listed on the ISO 8559 standard were obtained from the full body model.

\subsection{Data Postprocessing}

MS Excel was used to post process acquired anthropometric data to calculate the statistics stated on ISO 15535 standard for reporting anthropometric databases. These statistical values are as follows:

- Number of subjects,

- Minimum value of the variables,

- Maximum value of the variables,

- Arithmetic mean,

- Standard error of the mean,

- Standard Deviation,

- Coefficient of variation,

- Frequency Distribution,

- Skewness,

- Kurtosis,

- Percentiles, $1^{\text {st }}$ to $99^{\text {th }}$. Percentile values were calculated from the actual distribution of individual subjects in the sample rather than estimated from a theoretical Gaussian distribution using the sample mean and standard deviation: $1^{\text {st }}, 5^{\text {th }}, 10^{\text {th }}, 25^{\text {th }}, 50^{\text {th }}, 75^{\text {th }}, 90^{\text {th }}, 95^{\text {th }}$ and $99^{\text {th }}$. 
These statistical values were compared and found to be similar to those reported on DOD-HDBK-743A.

\subsection{Quality control and validation}

As stated in the ISO 15535 standard, once the mean and the standard deviation for each item is obtained, measurement data over \pm 3 SD was checked individually for accuracy. All data was normalized according with the variable mean and standard deviation.

Outliers from subjects with out of the common proportions in comparison with the rest of the population, were kept in the data set. On the contrary, if the outliers were originated from: (1) a deficient scan, or (2) an inadequate extraction algorithm, a correction was applied, if possible, otherwise the outlier was eliminated from data set.

To ensure that the anthropometric data was being collected and extracted consistently among all subjects, a validation process was conducted, based on the assumption that body dimensions are normally distributed [6].

A goodness of fit test based on the $x^{2}$ (chi-square) distribution was made for each one of the data sets, as follows:

1. Test data was divided into class intervals, following the Sturges formula, as shown in Eq.(3) :

$$
k=1.322 * \log N
$$

where:

$k=$ number of class intervals,

$N$ = number of subjects (sample size).

2. Determine the frequency of observations $O_{i}$, for each interval.

3. In order to use the chi square test, the number of samples per class intervals must be at least 5 [11], so the class intervals were modified manually to fulfill this requirement.

4. For each class interval, an expected frequency from the normal distribution $E_{i}$ is calculated.

5. The $\chi_{\exp }^{2}$ (Experimental chi square value) was calculated, using Eq.(4).

$$
\chi_{\exp }^{2}=\sum_{i=1}^{k} \frac{\left(O_{i}-E_{i}\right)^{2}}{E_{i}}
$$

As shown in Eq.(4), the greater the difference between observed and expected values, the greater the value of $\chi_{\text {exp }}^{2}$, so a $\chi_{\alpha, v}^{2}$ was calculated as a rejection criterion, where $\alpha$ is the significance of the test, and $v$ the degrees of freedom.

6. If $\chi_{\exp }^{2}<\chi_{0.05,4}^{2}$ the null hypothesis was accepted at the confidence level stated, implying that there would be enough statistical ton ensure the data set follows a normal distribution.

\section{Results}

\subsection{Quality Control}

Even though each scan was acquired and promptly inspected in situ, some scans were found to be defective when the data quality control was performed. The wrong acquisition was often related with bad positioning of the subject in the scanner, for example, Figure 3 a shows a subject whose arms were positioned behind the body, leading to a bad estimation of the breast point.

The most common error in positioning was related with the head. For the item Head Girth only 91 subjects were left from an original data set of 99. Like shown in Figure 3b some subjects bowed their heads, leading to a wrong approximation of head circumference. 


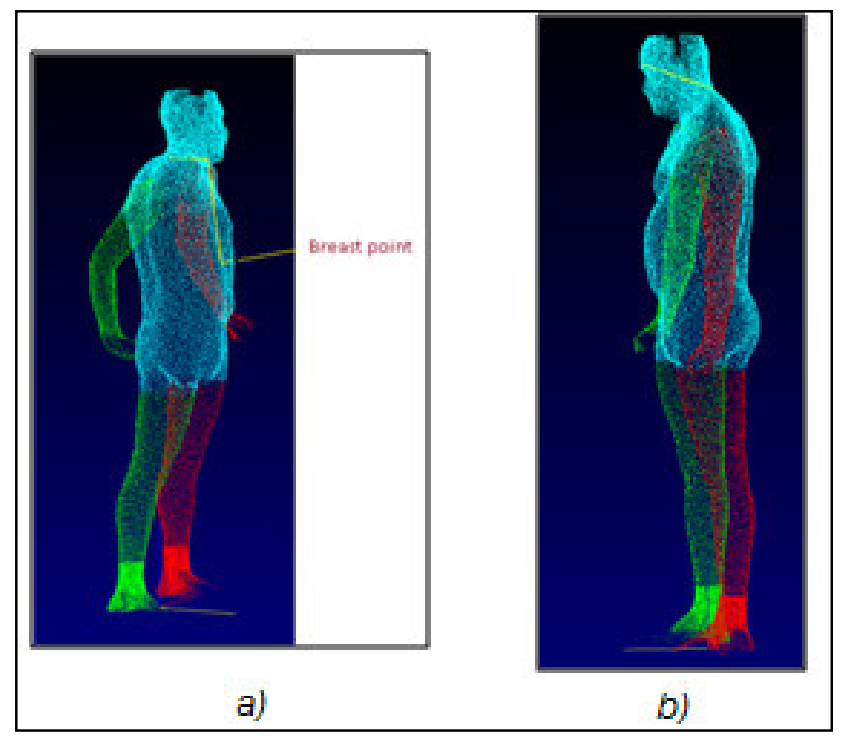

Fig.3. a) Wrong location of breast point due to poor positioning. b) Example of wrong calculation of head circumference.

Sometimes, even when the scans were acquired correctly, body landmarks were poorly located. In two of the scans, crotch point were misplaced in the body model, leading to wrong calculation of dimensions like crotch length, trunk circumference, inner leg length, among others. (See Figure 4a) This error was corrected manually relocating the landmark, as shown in Figure 4b.

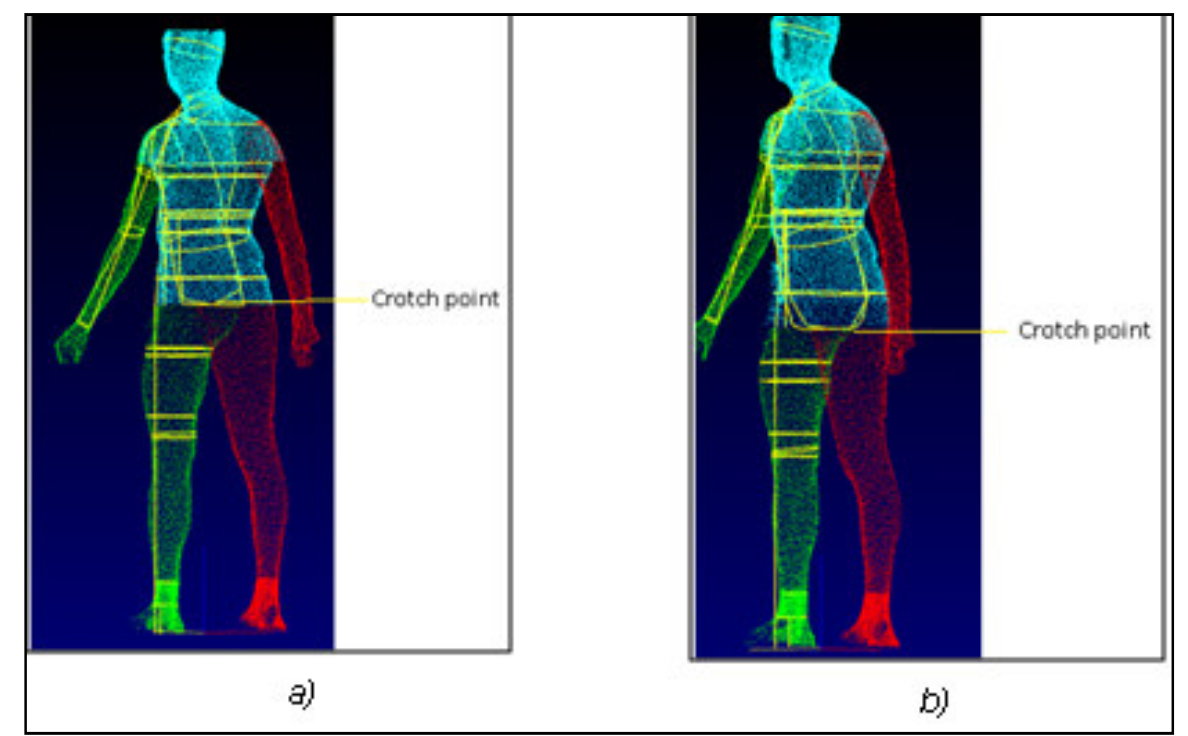

Fig.4. a) Example of wrong location of the crotch point by the automatic extraction software. b) Manually relocated crotch point.

\subsection{Statistical values}

Once the quality control was performed, the statistical values for each variable were calculated and compiled in tables. Due to the delicate nature of the study, privacy issues prevent the authors from showing the full anthropometric tables. Some of the statistical values are shown in table 2. For further information, please contact corresponding author. 
Table 2. Statistical values obtained for anthropometric variables

\begin{tabular}{|c|c|c|c|c|c|}
\hline & ISO 8559 & $N$ & C.V & MIN & MAX \\
\hline$\underline{2,1,1}$ & HEAD GIRTH [mm] & 91 & $3,1 \%$ & 522,0 & 610,0 \\
\hline$\underline{2,1,2}$ & NECK GIRTH [mm] & 96 & $6,2 \%$ & 333,0 & 452,0 \\
\hline$\underline{2,1,3}$ & NECK BASE GIRTH [mm] & 96 & $6,3 \%$ & 368,0 & 488,0 \\
\hline$\underline{2,1,4}$ & SHOULDER LENGTH [mm] & 96 & $10,4 \%$ & 92,0 & 177,0 \\
\hline$\underline{2,1,5}$ & SHOULDER WIDTH [mm] & 96 & $6,3 \%$ & 298,0 & 425,0 \\
\hline$\underline{2,1,6}$ & BACK WIDTH [mm] & 96 & $6,3 \%$ & 312,0 & 428,0 \\
\hline$\underline{2,1,7}$ & CHEST GIRTH [mm] & 96 & $7,5 \%$ & 840,0 & 1197,0 \\
\hline$\underline{2,1,9}$ & BUST WIDTH [mm] & 93 & $9,7 \%$ & 155,0 & 264,0 \\
\hline$\underline{2,1,11}$ & WAIST GIRTH [mm] & 95 & $10,0 \%$ & 671,0 & 1079,0 \\
\hline$\underline{2,1,12}$ & HIP GIRTH [mm] & 96 & $6,5 \%$ & 814,0 & 1112,0 \\
\hline$\underline{2,1,13}$ & UPPER ARM GIRTH [mm] & 96 & $9,1 \%$ & 243,0 & 383,0 \\
\hline$\underline{2,1,14}$ & ELBOW GIRTH [mm] & 96 & $6,6 \%$ & 219,0 & 307,0 \\
\hline$\underline{2,1,15}$ & WRIST GIRTH [mm] & 92 & $5,7 \%$ & 139,0 & 184,0 \\
\hline$\underline{2,1,18}$ & THIGH GIRTH [mm] & 96 & $7,6 \%$ & 406,0 & 603,0 \\
\hline$\underline{2,1,19}$ & MID THIGH GIRTH [mm] & 96 & $7,6 \%$ & 419,0 & 627,0 \\
\hline$\underline{2,1,20}$ & KNEE GIRTH [mm] & 95 & $5,7 \%$ & 328,0 & 455,0 \\
\hline$\underline{2,1,21}$ & LOWER KNEE GIRTH [mm] & 95 & $6,2 \%$ & 297,0 & 417,0 \\
\hline$\underline{2,1,22}$ & CALF GIRTH [mm] & 96 & $7,5 \%$ & 303,0 & 455,0 \\
\hline$\underline{2,1,23}$ & MINIMUM LEG GIRTH [mm] & 96 & $6,6 \%$ & 170,0 & 255,0 \\
\hline$\underline{2,2,2}$ & TRUNK LENGTH [mm] & 96 & $3,7 \%$ & 628,0 & 765,0 \\
\hline$\underline{2,2,3}$ & WAIST HEIGHT [mm] & 94 & $4,9 \%$ & 919,0 & 1139,0 \\
\hline$\underline{2,2,4}$ & HIP HEIGHT [mm] & 96 & $5,4 \%$ & 734,0 & 959,0 \\
\hline$\underline{2,2,5}$ & BODY RISE [mm] & 95 & $9,3 \%$ & 200,0 & 315,0 \\
\hline$\underline{2,2,6}$ & KNEE HEIGHT [mm] & 95 & $5,7 \%$ & 406,0 & 569,0 \\
\hline$\underline{2,2,7}$ & ANKLE HEIGHT [mm] & 96 & $11,8 \%$ & 53,0 & 95,0 \\
\hline$\underline{2,2,9}$ & SCYE DEPTH [mm] & 96 & $9,7 \%$ & 135,0 & 216,0 \\
\hline$\underline{2,2,10}$ & CERVICAL TO WAIST [mm] & 95 & $5,8 \%$ & 407,0 & 542,0 \\
\hline$\underline{2,2,11}$ & CERVICAL TO KNEE HOLLOW [mm] & 96 & $3,6 \%$ & 940,0 & 1116,0 \\
\hline$\underline{2,2,12}$ & CERVICAL HEIGHT [mm] & 96 & $3,9 \%$ & 1373,0 & 1662,0 \\
\hline$\underline{2,2,13}$ & CERVICAL TO BREAST POINT [mm] & 95 & $6,4 \%$ & 288,0 & 396,0 \\
\hline$\underline{2,2,14}$ & CERVICAL TO WAIST[mm] & 94 & $6,0 \%$ & 487,0 & 640,0 \\
\hline$\underline{2,2,15}$ & NECK SHOULDER POINT TO BREAST POINT [mm] & 95 & $7,4 \%$ & 211,0 & 306,0 \\
\hline$\underline{2,2,16}$ & FRONT WAIST LENGTH [mm] & 93 & $6,5 \%$ & 425,0 & 593,0 \\
\hline$\underline{2,2,17}$ & WAIST TO HIPS [mm] & 95 & $9,1 \%$ & 142,0 & 224,0 \\
\hline$\underline{2,2,18}$ & TRUNK CIRCUMFERENCE [mm] & 96 & $4,1 \%$ & 1480,0 & 1819,0 \\
\hline$\underline{2,2,19}$ & CROTCH LENGTH [mm] & 95 & $7,7 \%$ & 562,0 & 818,0 \\
\hline$\underline{2,2,20}$ & ARMSCYE GIRTH [mm] & 96 & $8,7 \%$ & 327,0 & 530,0 \\
\hline$\underline{2,2,21}$ & UPPER ARM LENGTH [mm] & 95 & $9,0 \%$ & 259,0 & 431,0 \\
\hline$\underline{2,2,22}$ & ARM LENGTH [mm] & 96 & $6,2 \%$ & 512,0 & 686,0 \\
\hline$\underline{2,2,23}$ & CERVICAL TO WRIST LENGTH [mm] & 96 & $5,2 \%$ & 691,0 & 897,0 \\
\hline$\underline{2,2,24}$ & UNDER ARM LENGTH [mm] & 96 & $7,0 \%$ & 393,0 & 555,0 \\
\hline$\underline{2,2,25}$ & OUTSIDE LEG LENGTH -OUTSEAM [mm] & 94 & $4,7 \%$ & 924,0 & 1103,0 \\
\hline$\underline{2,2,26}$ & THIGH LENGTH [mm] & 96 & $7,6 \%$ & 230,0 & 330,0 \\
\hline$\underline{2,2,27}$ & INSIDE LEG LENGTH [mm] & 96 & $5,6 \%$ & 654,0 & 869,0 \\
\hline
\end{tabular}




\subsection{Validation}

The Chi Square analysis for goodness of fit was applied for testing the normality of the variables. The significance $\alpha$ was chosen to be 0.05 , and the degrees of freedom $v$ were given by the number of class intervals minus the statistical values obtained from the experimental data: Mean, standard Deviation and number of class intervals.

For example, if the resultant number of class intervals for a data set came out to be seven, the degrees of freedom would be four, giving: $\chi_{\alpha, v}^{2}=\chi_{0.05,4}^{2}=9.48$.. If $\chi_{\exp }^{2}<9.48$. then the null hypothesis is accepted at the $95 \%$ confidence level.

It was found that all variables followed the normal distribution at the $95 \%$ confidence level, except for Knee Girth (ISO 8559 ref: 2.1.20). Since the experimental chi square value $\chi_{\text {exp }}^{2}$ was found to be higher than $\chi_{0.05,4}^{2}$ there was not enough evidence to support the hypothesis that data followed normality.

\section{Conclusions}

The first stage of the creation of an anthropometric database for colombian military personnel was accomplished acquiring and processing 44 anthropometric variables from 99 subjects (approximately one fifth of the total sample size calculated)..

The processing and compiling of anthropometric data was done according to the requirements stated in international standards. In order to assure the pertinence of the data collected and statistical values reported.

A quality control procedure for the data was implemented, which established that any data over \pm 3 SD must be individually reviewed. This method was proven to be very effective to identify defective body models and inadequately located landmarks.

A validation method was implemented in order to verify the consistency of the anthropometric data extraction procedures.

\section{Acknowledgements}

The research presented in this paper was funded by the Military Industry of Colombia (INDUMIL), through contract CIFI-4036.

\section{References}

1. United States Department of Defense, Military Handbook. Anthropometry of U.S military personnel (Metric). 1991: Nattick, MA.

2. Hwang, S. and C. Istook. Body measurement terminology used in the apparel industry. 2001.

3. Simmons, K., Body measurement techniques: a comparison of three-dimensional body scanning and physical anthropometric methods. 2001, North Carolina State University.

4. International Organization For Standardization, ISO 8559 Garment construction and anthropometric surveys - Body dimensions. 1989: Geneva-Switzerland.

5. International Organization For Standardization, ISO 7250-1 Basic human body measurements for technological design. 2008: Geneva, Switzerland.

6. International Organization For Standardization, ISO 15535. General requirements for stablishing anthropometric databases. 2006: Geneva, Switzerland.

7. International Organization For Standardization, ISO 20685 3-D scanning methodologies for internationally compatible anthropometric databases. 2005: Geneva-Switzerland.

8. $[\mathrm{TC}]^{2}, 3 D$ Body Scanner For Apparel, Health/Fitness/Medical, Entertainment/Gaming, and Virtual Worlds, $[\mathrm{TC}]^{2}$.

9. Radkte, R.A., NX12 Body Measurement System: Assessment of Risk for Photosensitive Seizures. 2006, Duke University Medical Center.

10. Allen, R., An Assessment of [TC]2 NX-16 Scanner Repeatability. 2008, Shape Analysis Limited.

11. Beckwith, T.G., R.D. Marangoni, and J.H. Lienhard, Mechanical measurements. 6th ed. 2007, Upper Saddle River, NJ: Pearson/Prentice Hall. xvi, 768 p. 